\title{
COX-2 rs20417 Polymorphism Is Associated with Stroke and White Matter Disease
}

\author{
Jamary Oliveira-Filho, MD, MS, $\mathrm{PhD},{ }^{*}+$ Ana C. P. Ornellas, MS, $\mathrm{PhD},{ }^{*}+$ \\ Cathy R. Zhang, PhD, $\neq$ Luciana M. B. Oliveira, MD, PhD, ${ }^{*}+$ Théo Araújo-Santos, $\mathrm{PhD}, \S$ \\ Valeria M. Borges, PhD, || Laís M. G. B. Ventura, MD, ${ }^{*}+$ Francisco J. F. B. Reis, MD, PhD, ${ }^{*}$ \\ Roque Aras, $\mathrm{MD}, \mathrm{PhD}^{*}$ André M. Fernandes, $\mathrm{MD},{ }^{*}$ Jonathan Rosand, $\mathrm{MD}, \mathrm{PhD}, \neq$ \\ Steven M. Greenberg, MD, PhD, $\ddagger$ Karen L. Furie, MD, MPH, $\uparrow$ \\ and Natalia S. Rost, MD, MPH†
}

\begin{abstract}
Background: To investigate the effect of COX-2 polymorphism and its product, prostaglandin E2 (PGE2), on stroke risk in an endemic area for Chagas disease. In a separate cohort, to investigate the effect of COX-2 polymorphisms on the total burden of cerebral white matter disease. Methods: Cases were outpatients with ischemic stroke; controls were stroke-free subjects from 2 outpatient clinics (heart failure and caregivers of a movement disorders clinic). We extracted DNA from total blood to investigate the rs20417 COX-2 polymorphism. Serologic tests (Enzime-linked immunosorbent assay) were performed to confirm Trypanosoma cruzi infection and to quantify PGE2 levels. In the Boston cohort, white matter hyperintensity volume (WMHv) was quantified on the admission brain magnetic resonance images of subjects with ischemic stroke, who also donated DNA for the COX-2 gene region analysis. Results: We studied 44 patients with stroke and 96 controls ( 46 with heart failure and 50 caregivers) in the Brazilian cohort; and 788 stroke patients (302 cardioembolic and 486 noncardioembolic) in the Boston cohort. In the Brazilian cohort, rs20417 polymorphism was associated with both stroke $\left(P=5 \times 10^{-6}\right)$ and decreased PGE2 levels $\left(P=4 \times 10^{-5}\right)$; similarly, Chagas was associated with stroke $\left(P=4 \times 10^{-3}\right)$ and decreased PGE2 levels $\left(P=7 \times 10^{-3}\right)$. In the Boston cohort, rs20417 polymorphism was associated with increased WMHv among noncardioembolic $(P=.037)$, but not among cardioembolic stroke patients. Conclusions: Variation in COX-2 gene is associated with both symptomatic and silent brain cerebrovascular disease. This candidate gene region should be tested in population-based samples. Key Words: Acute cerebral infarction-genetics of stroke-magnetic resonance imaging-Chagas disease—cyclooxygenase-prostaglandin E2.
\end{abstract}

(c) 2015 by National Stroke Association

\footnotetext{
From the *Stroke and Cardiomyopathy Clinics, Federal University of Bahia, Salvador, Brazil; +Instituto Nacional de Ciencia e Tecnologia em Doencas Tropicais (INCT-DT), Brazilian National Research Committee $(\mathrm{CNPq})$, Salvador, Brazil; $\ddagger$ Stroke Division, Massachusetts General Hospital, Boston, USA; §Universidade Federal do Oeste da Bahia (UFOB), Barreiras, Brazil; $\|$ Centro de Pesquisas Goncalo Moniz, Oswaldo Cruz Foundation-Bahia (CPqGM, FIOCRUZ-BA), Salvador, Brazil; and $\uparrow$ Department of Neurology, Brown University, Providence, USA.

Received January 23, 2015; revision received April 6, 2015; accepted April 12, 2015.
}

This work was supported, in part, by NIH grant R01NS063925 and a Brazilian National Committee in Research (CNPq) Productivity Grant (J.O.-F.).

Address correspondence to Jamary Oliveira-Filho, MD, MS, PhD, Rua Reitor Miguel Calmon, sem número, Instituto de Ciencias da Saude, sala 415, Salvador, Bahia 40110-100, Brazil. E-mail: jamary@ mail.harvard.edu.

$1052-3057 / \$$ - see front matter

(c) 2015 by National Stroke Association

http:/ /dx.doi.org/10.1016/j.jstrokecerebrovasdis.2015.04.018 
The human gene for cyclooxygenase-2 (COX-2) is located on chromosome 1q25.2-q25.3 and converts fatty acids into prostaglandins. The enzyme has 3 isoforms: COX-1, which is expressed in most tissues under basal conditions; COX-2, whose expression is induced in response to inflammatory stimuli; and $C O X-3$, which is an isoenzyme derived from COX-1. ${ }^{1}$ The expression of COX-2 is enhanced in chronic inflammatory diseases, including atherosclerosis and chronic infections. The rs20417 G>C COX-2 polymorphism has been associated with decreased gene expression and protection against coronary heart disease. ${ }^{2,3}$ However, no consistent association has been found with stroke, although a COX-2 inhibitor drug was withdrawn from the market because of increased stroke risk. ${ }^{4}$

Chagas disease is caused by Trypanosoma cruzi, a flagellate parasite acquired through contact with an insect vector, and it is a major cause of cardiac disease and stroke in South America. ${ }^{5} \mathrm{Up}$ to $8 \%$ of the South American population is seropositive for Chagas, but only $10 \%-30 \%$ of the infected individuals will present the symptomatic form of the disease. ${ }^{6}$ Over 300,000 individuals with the infection are estimated to live in the United States. ${ }^{7}$ Stroke is associated with Chagas independently of cardiomyopathy, ${ }^{8,9}$ and because Chagas serologic tests are not a routine part of stroke investigation, its prevalence is likely underestimated. The main product of the COX pathway, prostaglandin E2 (PGE2), is found in high levels in acute Chagas, ${ }^{10}$ and treatment with COX-2 inhibitors and subsequent inhibition of PGE2 synthesis can reduce cardiac damage during the acute phase of experimental Chagas disease. ${ }^{11}$ However, the exact role of COX-2 in chronic Chagas is unknown.

A total burden of white matter disease is detected on T2 magnetic resonance imaging (MRI) as white matter hyperintensity $(\mathrm{WMH})$, which is considered a marker of chronic small-vessel cerebrovascular disease. ${ }^{12}$ Although grouped under the term of "silent" brain pathology, $\mathrm{WMH}$ has been associated with cognitive decline and increased stroke risk. ${ }^{13}$ Several polymorphisms have been associated with the phenotype of WMH, which may identify important pathways in the pathophysiology of cerebrovascular diseases. ${ }^{14}$

\section{Aims}

We sought to determine the effect of the rs20417 COX-2 gene polymorphism on cerebrovascular disease in 2 separate cohorts. In a Brazilian cohort where Chagas is endemic, we investigated the effect of rs20417 polymorphism on stroke risk; in a Boston cohort, we investigated the effect of rs20417 and other COX-2 polymorphisms on WMH severity. Secondary aims in the Brazilian cohort were to determine whether there is a significant interaction between Chagas and the rs20417 polymorphism; and whether the rs20417 polymorphism and Chagas affect PGE2 serum levels.

\section{Methods}

\section{Brazilian Cohort}

\section{Patient Ascertainment}

The present study involved 2 cohorts (Brazil and Boston). In the Brazilian cohort, patients were recruited consecutively at a university-based outpatient clinic. Cases were patients with neuroimaging-confirmed ischemic stroke (IS) recruited from a reference stroke clinic at Hospital Universitario Professor Edgard Santos. Controls were stroke-free subjects from a heart failure (HF) clinic and caregivers from a movement disorders clinic. HF patients were part of a larger cohort study studying biomarkers and neuroimaging in Chagas, registered under Clinicaltrials.gov (identifier NCT01650792). We applied the Questionnaire for Verifying Stroke-Free Status $^{15}$ to exclude stroke from the HF and caregiver groups. IS was defined as an acute onset of a focal neurologic deficit lasting over 24 hours and confirmed by imaging (computed tomography or MRI). The present study was approved by the local ethics committee and all patients signed informed consent.

\section{Clinical Characteristics}

A standardized questionnaire was used to collect data on patient admission: demographic information such as age and sex, cerebrovascular risk factors such as systemic hypertension (defined as 2 outpatient blood pressure measurements above $140 / 90 \mathrm{~mm} \mathrm{Hg}$ or by previous antihypertensive medication use for this indication), dyslipidemia (defined as total serum cholesterol $>200 \mathrm{mg} / \mathrm{dL}$ or low-density lipoprotein $>100 \mathrm{mg} / \mathrm{dL}$ or high-density lipoprotein $<50 \mathrm{mg} / \mathrm{dL}$ or triglycerides $>150 \mathrm{mg} / \mathrm{dL}$ or by current statin or lipid-lowering medication use), diabetes mellitus (defined by a previous diagnosis or current medication use), atrial fibrillation detected on any previous electrocardiogram or Holter monitorization, and current smoking.

\section{Laboratory Analysis}

Genomic DNA was extracted from patients' blood using a Qiagen kit (QIAamp DNA Mini Kit). After extraction, a polymerase chain reaction (PCR) was performed using specific primers and after amplification, restriction fragment length polymorphism was conducted, with restriction enzymes specific for each polymorphism. To investigate the presence of the rs20417 polymorphism, primers CF8 (5' CCGCTTCCTTTGTCCATCAG $3^{\prime}$ ) and CR7 (5' GGCTGTATATCTGCTCTATATGC 3') with standard PCR conditions were used. The PCR product was subjected to restriction fragment length polymorphism 
using the restriction enzyme AciI. The PCR product consists of 306 base pairs, and the presence of the polymorphism will result in one band of 188 base pairs and another one of 118 base pairs, and can be visualized on $2 \%$ agarose gel on a ultraviolet transluminator.

PGE2 levels were estimated in plasma samples by enzyme-linked immunoassay, according to the manufacturer's instructions (Cayman Chemical).

\section{Boston Cohort}

\section{Patient Ascertainment}

Study subjects were recruited as part of an ongoing hospital-based study of patients with IS. Consecutive patients aged 18 years or older admitted to the Massachusetts General Hospital Stroke Unit, including those admitted directly to the emergency department or transferred to the emergency department from a referring hospital, between July 2000 and December 2013 were considered for enrollment. Patients were evaluated by a neurologist and imaged before diagnosis IS defined as either ${ }^{1}$ clinical stroke syndrome associated with radiographically proven infarct or $^{2}$ a fixed neurologic deficit persisting for more than 24 hours that was consistent with a vascular event but without evidence of demyelination or nonvascular disease. Only patients with axial T2-fluid attenuated inversion recovery sequences of quality suitable for quantification on cranial MRIs obtained less than 72 hours after admission for stroke were included in the analysis. Patients with symptoms of IS related to specific vascular disorders (such as vasculitis, subacute bacterial endocarditis, fibromuscular dysplasia, vasospasm due to subarachnoid hemorrhage, or cocaine abuse) or other known causes of WMH/stroke (such as CADASIL, MELAS, cranial radiotherapy, leukodystrophies, or mitochondrial disorders) were excluded. The institutional review board approved all aspects of this study, and informed consent was provided by all subjects or their medical proxy. Phenotyping of ischemic etiology was performed according to the Causative Classification of Stroke or TOAST (Trial of Org 10172 in Acute Stroke Treatment) Classification, as previously described. ${ }^{16,17}$ Patients with large-vessel atherosclerosis, small-vessel atherosclerosis, and other or undetermined causes of IS were grouped into a "noncardioembolic stroke" category and analyzed separately from patients with cardioembolic IS.

\section{Neuroimaging Analysis}

MRI scans were acquired on 1.5-T Signa scanners (GE Medical Systems, Milwaukee, WI) and converted from Digital Imaging and Communications in Medicine format to Analyze format, using MRIcro software (www.mricro. com). Using a previously published semiautomated method with high inter-rater reliability, axial T2-fluid attenuated inversion recovery sequences aligned with corresponding diffusion-weighted imaging sequences were used to create WMH volume (WMHv) maps, excluding acute ischemia, edema, and chronic territorial infarcts. ${ }^{18}$ The WMHv in the hemisphere unaffected by the acute stroke was doubled to calculate total WMHv, which was then normalized for head size.

\section{Genotyping}

Genotyping was performed on the Genome-Wide $\mathrm{Hu}-$ man SNP Array 6.0 (Affymetrix), Illumina Human610Quad v1.0 (Illumina), or HumanOmniExpress-12 v1.0 (Illumina) platforms. Standard quality control measures excluded improperly genotyped or rare single nucleotide polymorphisms and subjects who were (1) improperly genotyped, (2) related to another subject with higher genotyping rate, or (3) demonstrated to have non-European ancestry by principal component analysis with reference populations from the HapMap Project. Unobserved single nucleotide polymorphisms were imputed using Segmented HAPlotype Estimation and Imputation Tool (SHAPEIT) v2.5, IMPUTE2 v2.2.2, and phase 1 version 3 reference panels from the 1000 Genomes Project. All genetic analyses were performed using PLINK v1.07, including age, sex, and principal components 1 and 2 as covariates in association analysis of log-transformed WMHv.

\section{Statistical Analysis}

Descriptive statistics and regression models for the Brazilian cohort were performed using the Statistical Package for the Social Sciences (SPSS; IBM Corporation, Armonk) version 17.0. Normally distributed continuous variables were summarized as means and standard deviations, whereas categorical variables were expressed as proportions. All regression models were adjusted for age and sex. Multinomial regression was used to investigate the association between the rs20417 polymorphism (number of C alleles), Chagas (binary independent variable), and stroke (dependent variable). An interaction term between the rs20417 polymorphism and Chagas was used to test for multiplicative effect measure modification. We used log-transformed PGE2 levels because of the skewed distribution of PGE2 and applied linear regression to investigate the association between the rs20417 polymorphism (number of C alleles) and log-transformed PGE2 levels, also adjusted for age and sex. We reported crude $P$ values and considered values less than .05 after Bonferroni correction as statistically significant.

\section{Results}

Clinical characteristics of the Brazilian and Boston cohorts are shown in Table 1. In the Brazilian cohort, we studied 140 individuals, including 44 IS cases and 96 controls (50 caregivers and 46 stroke-free subjects with HF). Mean age was $64 \pm 11$ years, $84(60 \%)$ female. Among 
Table 1. Clinical characteristics of the Brazilian and Boston cohorts

\begin{tabular}{|c|c|c|c|c|}
\hline \multirow[b]{2}{*}{ Variable } & \multicolumn{3}{|c|}{ Brazilian cohort } & \multirow{2}{*}{$\begin{array}{c}\text { Boston cohort } \\
(\text { stroke } ; \mathrm{n}=788)\end{array}$} \\
\hline & Stroke $(\mathrm{n}=44)$ & Heart failure $(\mathrm{n}=46)$ & Controls $(\mathrm{n}=50)$ & \\
\hline Age, $y$, mean \pm SD & $65 \pm 13$ & $63 \pm 11$ & $65 \pm 9$ & $66 \pm 14$ \\
\hline Female sex, n (\%) & $21(48)$ & $26(57)$ & $35(70)$ & $298(38)$ \\
\hline Chagas disease, $\mathrm{n}(\%)$ & $19(43)$ & $22(48)$ & $5(10)$ & - \\
\hline \multicolumn{5}{|l|}{ rs20417 genotype } \\
\hline $\mathrm{GG}, \mathrm{n}(\%)$ & $5(11)$ & $9(20)$ & $34(68)$ & $20(3)$ \\
\hline $\mathrm{GC}, \mathrm{n}(\%)$ & $24(55)$ & $31(67)$ & $10(20)$ & $225(29)$ \\
\hline $\mathrm{CC}, \mathrm{n}(\%)$ & $15(34)$ & $6(13)$ & $6(12)$ & $543(69)$ \\
\hline Minor (C) allele frequency & .61 & .47 & .22 & .83 \\
\hline
\end{tabular}

Abbreviation: SD, standard deviation.

stroke cases, $18(40 \%)$ were cardioembolic and $26(60 \%)$ noncardioembolic. The Boston cohort had 788 IS patients, mean age $66 \pm 14$ years, 298 (38\%) female; 302 (38\%) were cardioembolic and 486 (62\%) noncardioembolic. Most etiologic phenotypic classification was performed using Causative Classification of Stroke, but in 264 (34\%) subjects the TOAST classification was used, with similar age distribution for subjects categorized according to each classification $(P=.094)$. When both classifications were available $(n=447)$, agreement between Causative Classification of Stroke and TOAST in cardioembolic category determination was $81 \%$. Distribution of rs20417 genotypes showed a predominance of the $C$ allele in the stroke groups $(61 \%$ in the Brazilian stroke group and $83 \%$ in the Boston cohort) and a predominance of the $G$ allele in the control group $(66 \%)$.

Table 2 shows the effect of the rs20417 COX-2 gene polymorphism and Chagas on the risk of stroke in the Brazilian cohort. We found a significant association between the rs20417 polymorphism and stroke (odds ratio, 6.97; 95\% confidence interval [CI], 3.02-16.07; $P=5.28 \times 10^{-6}$ ) and between Chagas and stroke (odds ratio, $\left.6.71 ; 95 \% \mathrm{CI}, 1.85-24.37 ; P=3.80 \times 10^{-3}\right)$. No significant multiplicative interaction between Chagas and the polymorphism was found $(P=.313)$. The association between the rs 20417 polymorphism and stroke remained significant when comparing the stroke group with both control groups (HF and caregivers) categorized as a single group (odds ratio, 3.51; 95\% CI, 1.86-6.63; $P=1.02 \times$ $\left.10^{-4}\right)$. Hardy-Weinberg equilibrium was met in all groups $(P=.319$ for the Brazilian stroke group, $P=.649$ for the Brazilian control group, and $P=.562$ for the Boston stroke cohort).

In Table 3, we show the effect of both the rs20417 polymorphism and Chagas on the main $\mathrm{COX}-2$ gene product, PGE2. Increasing $C$ allele count was associated with a significant decrease in PGE2 level (beta coefficient $=-.77$, standard error $=.19, P=4.1 \times 10^{-5}$ ); similarly, Chagas was also associated with decreased PGE2 level (beta coefficient $=-.76$, standard error $=.28, P=7.0 \times$ $\left.10^{-3}\right)$. However, no significant interaction was found between Chagas and the rs20417 polymorphism $(P=.431)$.

In the Boston cohort, 3 polymorphisms within the COX-2 gene region were significantly associated with WMHv: rs19545927, rs20417, and rs201231411 (Table 4). The only polymorphism associated with both cardioembolic and noncardioembolic stroke was rs199545927, whereas rs20417 and rs201231411 had significant association only with noncardioembolic stroke. The direction of the association of the rs20417 polymorphism was similar to the Brazilian cohort, with increasing $C$ allele count associated with increasing WMHv $(P=.037)$.

\section{Discussion}

The association between the COX-2 gene rs20417 polymorphism and stroke is controversial. Although adequately powered cohorts have suggested an association, ${ }^{19-23}$

Table 2. Multinomial regression predictors of stroke and heart failure in 140 patients from the Brazilian cohort, adjusted for age and sex

\begin{tabular}{clccc}
\hline Group & \multicolumn{1}{c}{ Variable } & Odds ratio & $95 \%$ CI & $P$ value \\
\hline \multirow{2}{*}{ Stroke versus Caregivers } & rs20417 C allele count & 6.97 for every additional allele & $3.02-16.07$ & $5.3 \times 10^{-6}$ \\
& Chagas disease & 6.71 & $1.85-24.37$ & $3.8 \times 10^{-3}$ \\
Heart failure versus caregivers & rs20417 C allele count & 3.25 for every additional allele & $1.55-6.79$ & $2.0 \times 10^{-3}$ \\
& Chagas disease & 8.00 & $2.46-26.03$ & $1.0 \times 10^{-3}$ \\
\hline
\end{tabular}

Abbreviation: CI, confidence interval.

$P=.313$ for COX-2/Chagas interaction. 
Table 3. Effects of COX-2 rs20417 polymorphism and Chagas disease on serum prostaglandin E2 level in 115 subjects from the Brazilian cohort, adjusted for age and sex

\begin{tabular}{cccc}
\hline & \multicolumn{3}{c}{ Standard } \\
Variable & Effect estimate & error & $P$ value \\
\hline $\begin{array}{c}\text { rs20417 minor } \\
\text { allele count } \\
(0,1, \text { or } 2)\end{array}$ & $\begin{array}{c}-.766 \text { for every } \\
\text { additional allele }\end{array}$ & .187 & $4.1 \times 10^{-5}$ \\
Chagas disease & -.755 & .282 & $7.0 \times 10^{-3}$ \\
\hline
\end{tabular}

$P=.431$ for COX-2/Chagas interaction

the most recent meta-analysis did not confirm prior findings. $^{2}$ One potential explanation is that the association is highly dependent on the interaction with environmental factors affecting expression of the gene. Experimental studies suggest that both acutely enhanced expression and chronic inhibition of the COX-2 pathway promotes neuronal damage. ${ }^{4,24,25}$ The major strengths of the present study are the investigation of the COX-2 gene pathway in a disease model (Chagas disease) where chronic inhibition of the pathway occurs; and the validation of the COX-2 gene pathway in acute and chronic vascular outcomes in 2 independent cohorts.

The major finding of the study is the evidence of association between the rs20417 polymorphism and stroke in the Brazilian cohort. The association was confirmed in both a case-control logistic regression model and a multinomial regression model. We also confirmed a previously demonstrated association between Chagas disease and stroke, $5,8,9$ which seems to be independent of cardiac disease and may be mediated by inflammatory mechanisms. Although it is known that triggers of inflammation, such as Chagas disease, influence COX-2 gene expression, we hypothesized that the interaction between these 2 factors would further increase stroke risk. However, we could not confirm this hypothesis, possibly due to insufficient statistical power.

Table 4. Effect of COX-2 gene polymorphisms on log-transformed white matter hyperintensity volume in 788 stroke patients from the Boston cohort (adjusted for age, sex, and principal components 1 and 2)

\begin{tabular}{|c|c|c|c|c|c|}
\hline SNP & Cohort & $\begin{array}{l}\text { Risk } \\
\text { allele }\end{array}$ & $\begin{array}{c}\text { Effect } \\
\text { estimate }\end{array}$ & $\begin{array}{l}\text { Standard } \\
\text { error }\end{array}$ & $\begin{array}{c}P \\
\text { value }\end{array}$ \\
\hline Rs199545927 & All & A & .247 & .124 & .047 \\
\hline Rs199545927 & Non-CE & A & .383 & .160 & .016 \\
\hline Rs20417 & All & $\mathrm{C}$ & .092 & .061 & .131 \\
\hline Rs20417 & Non-CE & $\mathrm{C}$ & .164 & .079 & .037 \\
\hline Rs201231411 & Non-CE & A & .339 & .149 & .023 \\
\hline
\end{tabular}

Abbreviations: CE, cardioembolic; SNP, single nucleotide polymorphism.

The non-CE cohort had 486 patients.
We further investigated the COX-2 gene pathway by measuring PGE2 serum levels in the Brazilian cohort and confirmed that both the rs20417 polymorphism and chronic Chagas disease were associated with lower PGE2 levels. Experimentally, both acutely elevated and chronically low PGE2 levels are associated with adverse outcomes in stroke. ${ }^{4,25}$ Treatment with prostaglandin agonist misoprostol decreased infarct size in a murine IS model. ${ }^{26}$ In a knock-out mouse model with acute Chagas disease infection, cardiac myocytes with low PGE2 levels had decreased survival when compared with wild-type mice with normal PGE2 levels. ${ }^{27}$ In human chronic cardiac Chagas disease, one study found that antimuscarinic acetylcholine receptor antibodies activate COX-2 gene expression and PGE2 production. ${ }^{28}$ Jointly, these data support the role of the $C O X-2$ gene pathway in stroke and Chagas disease, where the main effect of chronic low expression of the COX-2 gene pathway is to worsen both stroke risk and outcome.

We further explored the relevance of the COX-2 gene pathway in silent brain cerebrovascular disease and found new associations between 3 polymorphisms (rs19545927, rs20417, and rs201231411) within the locus of interest and WMHv. The finding is in agreement with a recent case-control Chinese study showing an association between $\mathrm{WMH}$ and 2 functional COX-2 gene polymorphisms (rs20417 and rs689466). ${ }^{23}$ White matter disease is a marker of small-vessel disease and increases with aging and cumulative burden of cerebrovascular risk factors. ${ }^{12,18}$ In addition, chronic inflammatory diseases such as lupus erythematous may trigger white matter disease without these cerebrovascular risk factors. $^{29,30}$

There are limitations to our study. The wide CIs and large effect size for stroke risk reflect the small sample size from the Brazilian cohort and increase the probability of a false-positive finding, highlighting the need for replication in a larger population-based sample to ensure generalizability of our findings. However, the results were robust in 2 separate statistical models, and the relevance of the COX-2 gene pathway in silent brain ischemia was confirmed in an independent, larger cohort. In the Brazilian cohort, controls were sampled from 2 separate groups that differed considerably in cerebrovascular risk factor burden, where patients with HF would be more similar to stroke patients than the caregiver group. If this were true (ie, controls are more similar to cases), then one would expect a decreased chance of finding significant association in this analysis, which is not the case. The Brazilian cohort is representative of regions of the world endemic for Chagas disease but may not generalize to southern Brazilian regions. We treated Chagas disease as a dichotomous variable based on previous data showing it affected stroke risk independently of cardiac disease, ${ }^{8,9}$ but cannot completely exclude other mechanisms of stroke in our patients with Chagas 
disease. Finally, the Boston cohort lacked a control group or information about Chagas status. However, we studied $\mathrm{WMH}$, a validated surrogate for chronic brain smallvessel disease, and the proportion of Chagas in the Boston sample is expected to be low.

\section{References}

1. Helgadottir A, Manolescu A, Thorleifsson G, et al. The gene encoding 5-lipoxygenase activating protein confers risk of myocardial infarction and stroke. Nat Genet 2004;36:233-239.

2. Li W, Xu J, Wang $X$, et al. Cyclooxygenase-2 (COX-2) G-765C is a protective factor for coronary artery disease but not for ischemic stroke: a meta-analysis. Atherosclerosis 2009;207:492-495.

3. Wang H, Fu Y, Liu D, et al. The COX-2 rs20417 polymorphism and risk of coronary artery disease: evidence from 17,621 subjects. Heart Lung Circ 2014;23:572-577.

4. Funk CD, FitzGerald GA. COX-2 inhibitors and cardiovascular risk. J Cardiovasc Pharmacol 2007;50:470-479.

5. Carod-Artal FJ. Stroke: a neglected complication of American trypanosomiasis (Chagas' disease). Trans R Soc Trop Med Hyg 2007;101:1075-1080.

6. Carod-Artal FJ, Gascon J. Chagas disease and stroke. Lancet Neurol 2010;9:533-542.

7. Bern C, Montgomery SP. An estimate of the burden of Chagas disease in the United States. Clin Infect Dis 2009;49:e52-e54.

8. Oliveira-Filho J, Viana LC, Vieira-de-Melo RM, et al. Chagas disease is an independent risk factor for stroke: baseline characteristics of a Chagas Disease cohort. Stroke 2005;36:2015-2017.

9. Paixao LC, Ribeiro AL, Valacio RA, et al. Chagas disease: independent risk factor for stroke. Stroke 2009; 40:3691-3694.

10. Corral RS, Guerrero NA, Cuervo H, et al. Trypanosoma cruzi infection and endothelin-1 cooperatively activate pathogenic inflammatory pathways in cardiomyocytes. PLoS Negl Trop Dis 2013;7:e2034.

11. Abdalla GK, Faria GE, Silva KT, et al. Trypanosoma cruzi: the role of PGE2 in immune response during the acute phase of experimental infection. Exp Parasitol 2008; 118:514-521.

12. Rost NS, Rahman RM, Biffi A, et al. White matter hyperintensity volume is increased in small vessel stroke subtypes. Neurology 2010;75:1670-1677.

13. Chutinet A, Rost NS. White matter disease as a biomarker for long-term cerebrovascular disease and dementia. Curr Treat Options Cardiovasc Med 2014;16:292.

14. Adib-Samii P, Rost N, Traylor M, et al. 17q25 Locus is associated with white matter hyperintensity volume in ischemic stroke, but not with lacunar stroke status. Stroke 2013;44:1609-1615.

15. Jones WJ, Williams LS, Meschia JF. Validating the Questionnaire for Verifying Stroke-Free Status (QVSFS) by neurological history and examination. Stroke 2001; 32:2232-2236.
16. Ay H, Benner T, Arsava EM, et al. A computerized algorithm for etiologic classification of ischemic stroke: the Causative Classification of Stroke System. Stroke 2007; 38:2979-2984.

17. Gordon DL, Bendixen BH, Adams HP Jr, et al. Interphysician agreement in the diagnosis of subtypes of acute ischemic stroke: implications for clinical trials. The TOAST Investigators. Neurology 1993;43:1021-1027.

18. Rost NS, Rahman R, Sonni S, et al. Determinants of white matter hyperintensity volume in patients with acute ischemic stroke. J Stroke Cerebrovasc Dis 2010;19: 230-235.

19. Kohsaka S, Volcik KA, Folsom AR, et al. Increased risk of incident stroke associated with the cyclooxygenase 2 (COX-2) G-765C polymorphism in African-Americans: the Atherosclerosis Risk in Communities Study. Atherosclerosis 2008;196:926-930.

20. Lee CR, North KE, Bray MS, et al. Cyclooxygenase polymorphisms and risk of cardiovascular events: the Atherosclerosis Risk in Communities (ARIC) study. Clin Pharmacol Ther 2008;83:52-60.

21. Yi XY, Zhou Q, Lin J, et al. Interaction between ALOX5AP-SG13S114A/T and COX-2-765G/C increases susceptibility to cerebral infarction in a Chinese population. Genet Mol Res 2013;12:1660-1669.

22. Chen GZ, Shan XY, Cheng GP, et al. Cyclooxygenase-2 genetic polymorphism and stroke subtypes in Chinese. J Mol Neurosci 2013;51:467-473.

23. Shan XY, Chen GZ, Cheng GP, et al. Cyclooxygenase 2 genetic polymorphism may increase the risk of developing leukoaraiosis in Chinese. J Mol Neurosci 2013; 51:461-466.

24. Cheng Y, Austin SC, Rocca B, et al. Role of prostacyclin in the cardiovascular response to thromboxane A2. Science 2002;296:539-541.

25. Liang X, Lin L, Woodling NS, et al. Signaling via the prostaglandin E(2) receptor EP4 exerts neuronal and vascular protection in a mouse model of cerebral ischemia. J Clin Invest 2011;121:4362-4371.

26. Li J, Liang X, Wang Q, et al. Misoprostol, an anti-ulcer agent and PGE2 receptor agonist, protects against cerebral ischemia. Neurosci Lett 2008;438:210-215.

27. Sharma J, Eickhoff CS, Hoft DF, et al. The absence of myocardial calcium-independent phospholipase A2gamma results in impaired prostaglandin E2 production and decreased survival in mice with acute Trypanosoma cruzi infection. Infect Immun 2013;81:2278-2287.

28. Ganzinelli S, Borda E, Joensen L, et al. Chagasic antibodies induce cardiac COX-2/iNOS mRNA expression with PGE2/NO production. Int J Cardiol 2009;134: 212-223.

29. Kozora E, Filley CM. Cognitive dysfunction and white matter abnormalities in systemic lupus erythematosus. J Int Neuropsychol Soc 2011;17:385-392.

30. Tektonidou MG, Varsou N, Kotoulas G, et al. Cognitive deficits in patients with antiphospholipid syndrome: association with clinical, laboratory, and brain magnetic resonance imaging findings. Arch Intern Med 2006; 166:2278-2284. 